\title{
Factors related to COVID-19 severity and mortality based on comparison between Henan and Zhejiang: an observational study
}

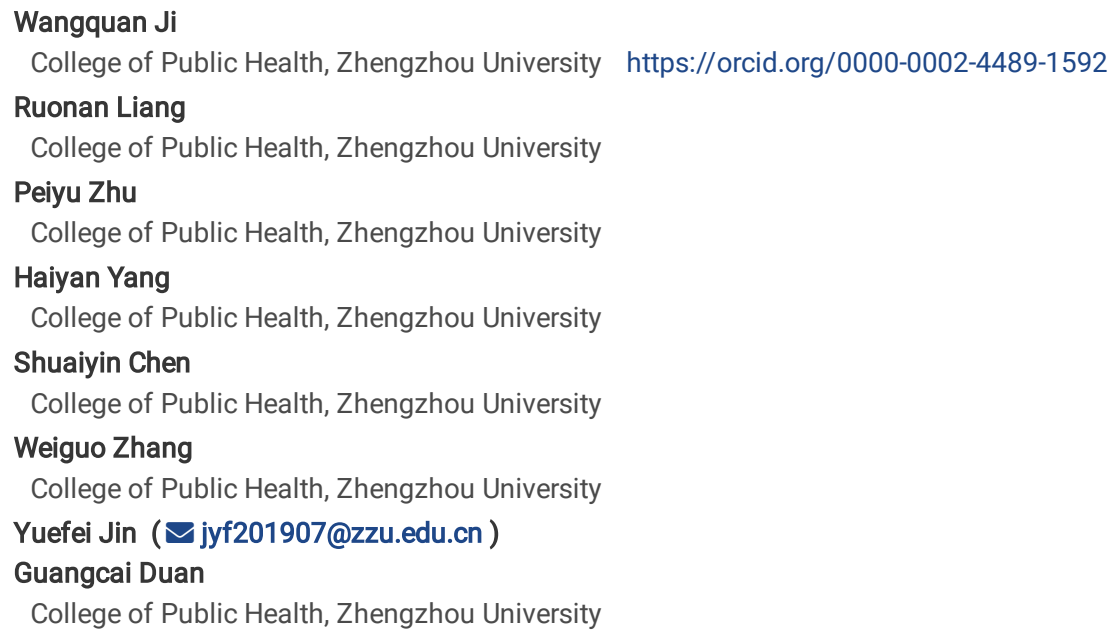




\section{Abstract}

Background: Coronavirus Disease 2019 (COVID-19) has spread across China and the world, since outbreak at the end of 2019. Although COVID-19 is generally considered as self-limiting, some of cases develop in to fatal respiratory restress, multiple organ failure and even sudden death. According to the data released by the provincial health commissions, there are obvious regional differences in COVID-19 mortality. To explore possible factors of COVID-19 related severity and mortality in laboratory-confirmed cases from Henan and Zhejiang.

Methods: We collected the public data released by the government. We analyzed and compared the possible factors, including initial symptoms, gender, age, place of residence, time interval from onset to diagnosis, epidemiological history and the grade of hospital for patient's treatment in laboratory-confirmed cases. Student's t-test, Chi-square $\left(c^{2}\right)$ test, Cochran-Mantel-Haenszel (CMH) test and Mann-Whitney U rank sum test were used complied with the applicable conditions. Statistical test was performed with a level of significance $a=0.05$.

Results: Death cases in Henan were mainly from male, elderly infected persons and those who had a history of contact with patients and underlying basic diseases. Henan had the higher proportion of cases with fever and the lower proportion of asymptomatic infection, and had higher proportions of imported male cases with a contact history, and elderly cases living in rural areas, relative to Zhejiang. The time interval of confirmed cases in rural areas of Henan was 0.8 day longer than that in Zhejiang. Hospitals grade for patients' treatment in Henan was lower than Zhejiang.

Conclusions: Our findings suggest that initial symptoms, age, place of residence, gender, the time interval from onset to diagnosis and the grade of designated hospital for patient's treatment are possible factors behind COVID-19 related severity or mortality. This study will provide useful information for public health authorities to develop disease prevention strategies.

\section{Introduction}

At the end of 2019, an emerging infectious disease named as Coronavirus Disease 2019 (COVID-19) occurred in several local hospitals of Wuhan, Hubei Province, China(1). Since then, continuous human to human transmissions were observed in Hubei and its outsides leading to more than 80,000 infected persons across mainland China(2). Based on clinical manifestations, blood tests and chest radiographs, this disease was initially considered as viral respiratory illness by clinicians(3). Subsequently, a novel human coronavirus named as severe acute respiratory syndrome (SARS) coronavirus 2 (SARS-CoV-2) was identified from the bronchoalveolar lavage fluid (BALF) of COVID-19 patients(4,5). COVID-19 is generally considered as a type of self-limiting infectious disease. Most of patients are mild, whereas some of patients develop into fatal respiratory distress posing a great threat to human life(4). To control the epidemic of COVID-19, a variety of interventions, such as containment strategy and emergency response were rapidly started by Chinese government. However, as lack of specific antiviral protection and vaccines available for COVID-19, more than 3,000 people died from this infectious disease in mainland China until March 09, 2020(2).

Epidemiological studies suggest that the most common factors related to COVID-19 severity or mortality are older age and concomitant disease(6). Of 44,672 laboratory confirmed cases, the total case-fatality rate is $2.3 \%$ (male: $2.8 \%$; female: $1.7 \%$ ), In contrast, the case-fatality rate of patients over 80 -year-old is as high as $14.8 \%$, and the case-fatality rate of patients with cardiovascular disease, diabetes, chronic respiratory disease, hypertension and cancer is $10.5 \%, 7.3 \%$, $6.3 \%, 6.0 \%$ and $5.6 \%$, respectively(6). Additionally, the case-fatality of critical cases is more than $50 \%$, which is higher than SARS(7). According to the data released by the provincial Health Commissions, there are obvious regional differences in COVID-19 mortality(8). For example, Guangdong, Henan and Zhejiang had similar number of cases $(1331,1262,1173)$ until 19 February, 2020 , but the case-fatality rates were quite different $(0.4 \%, 1.5 \%, 0.0 \%)(8)$. To figure out possible factors of COVID-19 related severity and mortality, clinical features, epidemiological characteristics and designated hospitals of Henan and Zhejiang were analyzed and compared in this study. This study will provide useful information for public health authorities to develop disease prevention strategies.

\section{Materials And Methods}

\section{Data source}

All data was collected from public data released by the government, including National Health Committee of the People's Republic of China (http://www.nhc.gov.cn/), Health Commission of Henan Province (http://www.hnwsjsw.gov.cn/Template/fangkong.shtml), Health Commission of Zhejiang Province (http://www.zjwjw.gov.cn/col/col1202101/index.html) and other official websites of urban areas.

By February 19, 2020, 1262 laboratory-confirmed cases, including 19 deaths (3 deaths with unknown information) were collected in Henan Province, while 1105 laboratory confirmed cases were collected from Zhejiang Province and no deaths was reported.

\section{Data Extraction And Entry}

Forms used for web data extraction were made by ourselves. Before conducting data extraction, all research participants need to undergo unified training. The main extracted information was gender, age, place of residence, time interval from onset to diagnosis, epidemiological contact history, etc. Data were entered twice into the software Microsoft Office Excel 2016 (Microsoft Corporation, Washington, USA) to correct for typing errors.

\section{Statistical analysis}

All statistical analyses were evaluated with SPSS 25.0 software (SPSS Inc. IBM, Chicago, IL, USA). Quantitative variables were expressed as mean \pm standard deviations (SD). Qualitative variables were expressed as ratios or rates (\%). Rank variables were expressed as mean rank. Comparisons between quantitative 
variables were tested by using Student's t-test. The Chi-square $\left(\chi^{2}\right)$ test was used to compare the statistical differences between qualitative variables. The Cochran-Mantel-Haenszel (CMH) test was applied to analyze stratified categorical data. Grading variables were analyzed by Mann-Whitney $U$ rank sum test. Statistical test was performed with a level of significance $a=0.05$.

\section{Results}

\section{An overview of COVID-19 epidemic in Henan and Zhejiang}

As shown in Fig. 1, Henan is located in the central China with a population of 106.06 million, and Zhejiang is located in the east China with a population of 53.37 million. Henan borders with Hubei, while Zhejiang is about $200 \mathrm{~km}$ from Hubei. On January 21, 2019, the two provinces started to announce information about epidemic situation, and the epidemic trend of these two provinces was very similar after analysis. The cumulative number of laboratory-confirmed cases in these two provinces began to increase rapidly after January 27 . The existing number of laboratory-confirmed cases in both provinces had declined since February 7, whereas Zhejiang was less than that in Henan after February 10 (Additional file 1: Figure.1). For new laboratory-confirmed cases (Figure.2A), the number of cases in both provinces arose rapidly after January 24 and declined after February 3 . The epidemic appeared two peaks on January 24 (132 confirmed cases) and February 3 (105 confirmed cases) in Zhejiang and one peak on February 3 (109 confirmed cases) in Henan. As shown in Figure.2B, the cumulative cure rate (\%) in Zhejiang was higher than that in Henan. China CDC data(6) showed that COVID-19 related mortality mainly came from severe and critical cases and the case-fatality rate of critical cases was as high as $49 \%$. As shown in Figure.2C, the severe rate of both provinces rapidly increased at the early phase and Henan reached a peak (33.33\%) on January 23. Since January 27, the severe rates of both provinces began to decline and fluctuated around $10 \%$. Notably, the number of critical cases in Henan was higher than that in Zhejiang since February 1, and the highest number of critical cases in Henan and Zhejiang was 38 and 31, respectively (Figure.2D). The number of critical cases in Henan and Zhejiang began to decrease after February 12 and 14 , respectively. Taken together, higher severe rate at early epidemic phase, lower cure rate and higher number of critical cases may predict more deaths in Henan.

\section{General Features Of Death Cases In Henan Province}

Since February 5, deaths continued to be reported in Henan, but no deaths in Zhejiang until February 19. A total of 19 deaths was announced in Henan by February 19, and the general features of 16 death cases ( 3 deaths with unknown information) were placed in Table 1 . The average age of these 16 deaths was (72 \pm 12.42 ) years old with a gender ratio (male/female) of 5:3, and $93.75 \%$ (15), 56.25\% (9), 31.25\% (5), 6.25\% (1) of them were $\geq 60$-year-old, $\geq 70$-year-old, $\geq 80$-year-old, $<60$-year-old, respectively. The common symptom of 9 cases with known information was fever (6, 66.7\%). 6 cases (37.5\%) lived in rural areas, and 9 cases (56.25\%) lived in urban areas. The time interval from onset to hospitalization was $(2.58 \pm 2.71)$ days, and the time interval from onset to diagnosis was $(6.77 \pm 2.89)$ days, and time interval from onset to death was (12.08 \pm 5.95$)$ days (Figure.3). There were 3 imported cases (18.75\%) coming from Wuhan, Hubei, and 10 (62.50\%) non-imported cases including 8 confirmed cases $(80.00 \%)$ having a history of close contact with patients. Additionally, underlying basic diseases, such as respiratory disorders, hypertension, cardiovascular diseases, diabetes and cancer were reported in 8 cases with detail information. 
Table 1

General features of death cases in Henan Province

\begin{tabular}{|c|c|c|c|c|c|c|c|c|c|c|c|c|}
\hline ID & $\begin{array}{l}\text { Gender } \\
(\mathrm{F} / \mathrm{M})\end{array}$ & Age & $\begin{array}{l}\text { Place of } \\
\text { residence }\end{array}$ & Symptoms & $\begin{array}{l}\text { Days from } \\
\text { onset to } \\
\text { admission }\end{array}$ & $\begin{array}{l}\text { Days } \\
\text { from } \\
\text { onset to } \\
\text { diagnosis }\end{array}$ & $\begin{array}{l}\text { Days } \\
\text { from } \\
\text { onset } \\
\text { to } \\
\text { death }\end{array}$ & $\begin{array}{l}\text { Imported } \\
\text { case? }\end{array}$ & $\begin{array}{l}\text { Wuhan } \\
\text { travel } \\
\text { history? }\end{array}$ & $\begin{array}{l}\text { Hubei } \\
\text { travel } \\
\text { history? }\end{array}$ & $\begin{array}{l}\text { Case } \\
\text { contact } \\
\text { history? }\end{array}$ & Basic dis€ \\
\hline 1 & Male & $\begin{array}{l}70 \sim \\
80\end{array}$ & Rural & Cough/asthma & 7 & 10 & 10 & Yes & Yes & Yes & UN & Chronic br \\
\hline 2 & Male & $80 \sim$ & Rural & $\begin{array}{l}\text { Fever/cough/sputum } \\
\text { /asthma }\end{array}$ & 1 & 4 & 5 & Yes & Yes & Yes & UN & Chronic br \\
\hline 3 & Male & $\begin{array}{l}60 \sim \\
70\end{array}$ & Rural & UN & UN & 7 & 8 & No & No & No & Yes & Diabetes/ \\
\hline 4 & Female & $80 \sim$ & Urban & UN & 1 & 4 & 8 & No & No & No & Yes & UN \\
\hline 5 & Female & $\begin{array}{l}70 \sim \\
80 \sim\end{array}$ & Urban & UN & UN & UN & UN & No & No & No & Yes & UN \\
\hline 6 & Male & $\begin{array}{l}70 \sim \\
80\end{array}$ & Urban & UN & UN & UN & UN & No & No & No & Yes & UN \\
\hline 7 & Female & $80 \sim$ & Urban & UN & 6 & 8 & 19 & No & No & No & Yes & Diabetes/। \\
\hline 8 & Male & $\begin{array}{l}70 \sim \\
80\end{array}$ & Urban & $\begin{array}{l}\text { Diarrhea/nausea/ } \\
\text { vomiting }\end{array}$ & 1 & 10 & 19 & UN & UN & UN & UN & Hypertens \\
\hline 9 & Male & $\begin{array}{l}60 \sim \\
70\end{array}$ & Rural & Fever & 8 & 9 & 11 & No & No & No & Yes & Lung canc \\
\hline 10 & Male & $\begin{array}{l}60 \sim \\
70\end{array}$ & UN & UN & UN & UN & UN & UN & UN & UN & UN & UN \\
\hline 11 & Male & $\begin{array}{l}60 \sim \\
70\end{array}$ & Rural & Fever & 2 & 4 & 19 & Yes & Yes & Yes & UN & UN \\
\hline 12 & Male & $\begin{array}{l}\text { Under } \\
60\end{array}$ & Urban & Fever & 1 & 10 & 20 & No & No & No & UN & UN \\
\hline 13 & Male & $\begin{array}{l}60 \sim \\
70\end{array}$ & Urban & Constipation & 1 & 8 & 9 & UN & UN & UN & UN & UN \\
\hline 14 & Female & $\begin{array}{l}60 \sim \\
70\end{array}$ & Urban & UN & 1 & 8 & 21 & No & No & No & UN & Hypertens \\
\hline 15 & Female & $80 \sim$ & Rural & Fever/cough & 1 & 5 & 8 & No & No & No & Yes & UN \\
\hline 16 & Female & $80 \sim$ & Urban & Fever & 1 & 1 & UN & No & No & No & Yes & Hypertens \\
\hline
\end{tabular}

\section{Presentation of initial symptoms of laboratory-confirmed cases in Henan and Zhejiang}

Due to individual differences in different patients or factors such as viral virulence and quantity, the initial symptoms of patients after infection often differ. The different initial symptoms may indicate different disease progression and outcomes. Based on the above considerations, we briefly describe and compare the initial symptoms of patients in the two provinces. We finally collected 939 cases in Zhejiang province and 676 cases in Henan province with the data of initial symptoms. As is shown in Figure.4, there were many differences in the initial symptoms of patients between the two provinces. Markedly, the results showed that $87.4 \%$ of the patients in Henan had fever in their initial symptoms, compared with Zhejiang Province $(75.1 \%)$, with significant significances $(P<$ 0.001). However, $37.8 \%$ of patients in Zhejiang Province had more cough than that in Henan Province (17.2\%), with significant significances $(P<0.001)$. Most other initial symptoms are statistically different (Table 2). It was worth noting that $2.1 \%$ of infected persons were asymptomatic in Henan, while $7.2 \%$ of patients were asymptomatic in Zhejiang $(P<0.001)$. However, potential information bias should be considered here. For instance, most of the data reported by Henan didn't include CT imaging performance, and fever and asymptomatic were commonly information reported by both provinces. Herein, the above data indicate that Henan has a higher proportion of patients with fever and lower proportion of patients with asymptomatic. 
Table 2

Presentation of initial symptoms of laboratory-confirmed cases in Henan and Zhejiang

\begin{tabular}{|c|c|c|c|c|}
\hline Province and symptoms & Zhejiang $(n=939)$ & Henan $(n=676)$ & $x^{2}$ & $P$ \\
\hline \multicolumn{5}{|l|}{ Fever } \\
\hline Yes & 705(75.1\%) & $591(87.4 \%)$ & \multirow[t]{2}{*}{37.797} & \multirow[t]{2}{*}{$<0.001$} \\
\hline No & $234(24.9 \%)$ & $85(12.6 \%)$ & & \\
\hline \multicolumn{5}{|l|}{ Cough } \\
\hline Yes & $355(37.8 \%)$ & $116(17.2 \%)$ & \multirow[t]{2}{*}{81.101} & \multirow[t]{2}{*}{$<0.001$} \\
\hline No & $584(62.2 \%)$ & $560(82.2 \%)$ & & \\
\hline \multicolumn{5}{|l|}{ Expectoration } \\
\hline Yes & $83(8.8 \%)$ & $24(3.69 \%)$ & \multirow[t]{2}{*}{17.772} & \multirow[t]{2}{*}{$<0.001$} \\
\hline No & $856(91.2 \%)$ & $652(96.4 \%)$ & & \\
\hline \multicolumn{5}{|l|}{ Rhinobyonh or rhinorrhea } \\
\hline Yes & $41(4.4 \%)$ & $17(2.5 \%)$ & \multirow[t]{2}{*}{3.892} & \multirow[t]{2}{*}{0.049} \\
\hline No & $898(95.6 \%)$ & $659(97.5 \%)$ & & \\
\hline \multicolumn{5}{|l|}{ Sore throat } \\
\hline Yes & $87(9.3 \%)$ & $26(3.8 \%)$ & \multirow[t]{2}{*}{17.737} & \multirow[t]{2}{*}{$<0.001$} \\
\hline No & $852(90.7 \%)$ & $650(96.2 \%)$ & & \\
\hline \multicolumn{5}{|c|}{ Chest pain or shortness of breath } \\
\hline Yes & $26(2.8 \%)$ & $27(4.0 \%)$ & \multirow[t]{2}{*}{1.859} & \multirow[t]{2}{*}{0.173} \\
\hline No & $913(97.2 \%)$ & $649(96.0 \%)$ & & \\
\hline \multicolumn{5}{|l|}{ Muscle ache or fatigue } \\
\hline Yes & $125(13.3 \%)$ & $61(9.0 \%)$ & \multirow[t]{2}{*}{7.093} & \multirow[t]{2}{*}{0.008} \\
\hline No & $814(86.7 \%))$ & 615(91.0\%) & & \\
\hline \multicolumn{5}{|l|}{ Headache or dizziness } \\
\hline Yes & $95(10.1 \%)$ & $22(3.3 \%)$ & \multirow[t]{2}{*}{27.547} & \multirow[t]{2}{*}{$<0.001$} \\
\hline No & $844(89.9 \%))$ & $654(96.7 \%)$ & & \\
\hline \multicolumn{5}{|l|}{ Nausea and vomiting } \\
\hline Yes & $19(2.0 \%)$ & $12(1.8 \%)$ & \multirow[t]{2}{*}{0.129} & \multirow[t]{2}{*}{0.720} \\
\hline No & $920(98.0 \%))$ & $664(98.2 \%)$ & & \\
\hline \multicolumn{5}{|l|}{ Diarrhoea } \\
\hline Yes & $20(2.1 \%)$ & $4(0.6 \%)$ & 6.325 & 0.012 \\
\hline No & 919(97.9\%)) & 672(99.4\%) & & \\
\hline Abnormal CT image & & & & \\
\hline Yes & $263(28.0 \%)$ & $2(0.3 \%)$ & 220.070 & $<0.001$ \\
\hline No & $676(72.0 \%))$ & 674(99.7\%) & & \\
\hline Asymptomatic & & & & \\
\hline Yes & $68(7.2 \%)$ & $14(2.1 \%)$ & 21.804 & $<0.001$ \\
\hline No & $871(92.8 \%))$ & $662(97.9 \%)$ & & \\
\hline
\end{tabular}

\section{Overall Comparison Of Laboratory-confirmed Cases In Henan And Zhejiang}

Of total confirmed cases, the proportion of male and female was 50.5\%, 49.5\% in Zhejiang, and 54.4\%, 45.6\% in Henan. The proportion of male in Henan was higher, but the difference had no statistically significance $(P>0.05)$. The mean age of the confirmed cases in Zhejiang was (47.25 \pm 15.30$)$, which was higher than that in Henan $(44.31 \pm 16.06)$ with significant difference $(P<0.001)$. Next, we performed a stratified analysis of age distribution. The proportion of $19 \sim$ 35 -year-old confirmed cases (28.9\%) in Henan was higher than that in Zhejiang (20.7\%) $(P<0.001)$. The proportion of $36 \sim 59$-year-old $(56.2 \%)$ and $\geq 60$-year- 
old confirmed cases (20.3\%) in Zhejiang was higher than that in Henan (51.8\%, 15.1\%), with significant significances ( $P=0.033, P=0.001)$ (Additional file 1: Table 1). $79.6 \%$ of confirmed cases lived in urban areas of Zhejiang, which was higher than that in Henan (49.7\%) with a significant difference $(P<0.001)$. Compared with Zhejiang (46.3\%), the proportion of imported cases in Henan (54.4\%) was higher, and the difference had a statistically significance ( $P<0.001)$. $41.9 \%$ and $44.0 \%$ of the confirmed cases in Zhejiang had Hubei and Wuhan travel history, which was lower than that in Henan (57.4\%, 54.6\%). $85.5 \%$ of the confirmed cases in Zhejiang had a history of contact with case, which was lower than that in Henan (91.6\%). The above differences were statistically significant $(P<0.001)$. The time interval from onset to diagnosis in Henan was $(7.35 \pm 4.20)$ days, which was 0.4 day longer than that in Zhejiang $(6.95 \pm 4.09)$ days, but the difference was not statistically significant $(P>0.05)$ (Table 3$)$. These results suggest the laboratory-confirmed cases in Henan mainly come from young, rural and imported infected persons with a Hubei or Wuhan travel history, relative to Zhejiang.

Table 3

Comparison of laboratory-confirmed cases in Henan and Zhejiang

\begin{tabular}{|c|c|c|c|c|}
\hline Province and variables & Zhejiang n (\%) & Henan $\mathrm{n}(\%)$ & $x^{2 / t}$ & $P$ \\
\hline \multicolumn{5}{|l|}{ Gender } \\
\hline Male & $542(50.5 \%)$ & $686(54.4 \%)$ & \multirow[t]{2}{*}{3.603} & \multirow[t]{2}{*}{0.058} \\
\hline Female & $532(49.5 \%)$ & $575(45.6 \%)$ & & \\
\hline Total & $1074(100 \%)$ & $1261(100 \%)$ & & \\
\hline \multicolumn{5}{|l|}{ Age (year) } \\
\hline$(0-18)$ & $30(2.8 \%)$ & $53(4.2 \%)$ & \multirow[t]{4}{*}{29.781} & \multirow[t]{4}{*}{$<0.001$} \\
\hline$(19-35)$ & $220(20.7 \%)$ & $363(28.9 \%)$ & & \\
\hline$(36-59)$ & $604(56.2 \%)$ & $652(51.8 \%)$ & & \\
\hline$(\geq 60)$ & $218(20.3 \%)$ & 190(15.1\%) & & \\
\hline Total & $1074(100 \%)$ & $1258(100 \%)$ & & \\
\hline \multicolumn{5}{|l|}{ Place of residence } \\
\hline Urban areas & $848(79.6 \%)$ & $622(49.7 \%)$ & \multirow[t]{2}{*}{222.483} & \multirow[t]{2}{*}{$<0.001$} \\
\hline Rural areas & $217(20.4 \%)$ & $630(50.3 \%)$ & & \\
\hline Total & $1065(100 \%)$ & $1252(100 \%)$ & & \\
\hline \multicolumn{5}{|l|}{ Imported case } \\
\hline Yes & $483(46.3 \%)$ & $673(54.4 \%)$ & \multirow[t]{2}{*}{14.839} & \multirow[t]{2}{*}{$<0.001$} \\
\hline No & $559(53.6 \%)$ & $563(45.6 \%)$ & & \\
\hline Total & $1042(100 \%)$ & $1236(100 \%)$ & & \\
\hline \multicolumn{5}{|l|}{ From Hubei Province } \\
\hline Yes & $392(41.9 \%)$ & $596(57.4 \%)$ & \multirow[t]{2}{*}{46.914} & \multirow[t]{2}{*}{$<0.001$} \\
\hline No & $543(58.1 \%)$ & $443(42.6 \%)$ & & \\
\hline Total & $935(100 \%)$ & $1039(100 \%)$ & & \\
\hline \multicolumn{5}{|l|}{ From Wuhan city } \\
\hline Yes & $404(44.0 \%)$ & $567(54.6 \%)$ & \multirow[t]{2}{*}{21.753} & \multirow[t]{2}{*}{$<0.001$} \\
\hline No & $514(56.0 \%)$ & $472(45.4 \%)$ & & \\
\hline Total & $918(100 \%)$ & $1039(100 \%)$ & & \\
\hline \multicolumn{5}{|l|}{ Contact cases } \\
\hline Yes & $511(85.5 \%)$ & $514(91.6 \%)$ & \multirow[t]{2}{*}{10.779} & \multirow[t]{2}{*}{0.001} \\
\hline No & $87(14.5 \%))$ & $47(8.4 \%)$ & & \\
\hline Total & $598(100 \%)$ & $561(100 \%)$ & & \\
\hline Age (year) & $47.25 \pm 15.30$ & $44.31 \pm 16.06$ & 4.505 & $<0.001$ \\
\hline Time interval (day) & $6.95 \pm 4.09$ & $7.35 \pm 4.20$ & 1.871 & 0.061 \\
\hline
\end{tabular}

\section{Comparison of laboratory confirmed cases in Henan and Zhejiang after adjusting for} gender 
Latest studies suggest that the male confirmed cases may have a higher case-fatality rate $(6,9)$. Nevertheless, the gender is a common confounding factor for other research factors. Than the stratified $\chi^{2}$ test was performed. $60.3 \%$ of male were imported cases in Henan, which were higher than that in Zhejiang (49.0\%) with a significant difference $(P<0.001)$. The proportion of male confirmed cases with a case contact history in Henan (92.5\%) was higher than that in Zhejiang (83.1\%), with a statistically significant difference $(P=0.001)$. The differences of age in male/female confirmed cases between Henan and Zhejiang had statistically significance $(P<0.05)$. Compared to Zhejiang, the proportion of male/female confirmed cases living in rural areas of Henan was higher with statistically significant differences $(P<0.001)$. The proportion of male confirmed cases with a Hubei or Wuhan travel history was higher than that in Zhejiang, and all differences had statistically significances $(P<0.001)$. The time interval from onset to diagnosis of confirmed cases in Henan was higher than that in Zhejiang whatever male or female, but had no statistically significance $(P>0.05)$ (Table 4). Above results indicate that Henan has a higher proportion of male cases coming from rural areas, or imported cases with epidemiological history, relative to Zhejiang. 
Table 4

Comparison of laboratory-confirmed cases in Henan and Zhejiang after adjusting for gender

\begin{tabular}{|c|c|c|c|c|c|c|c|c|}
\hline \multirow[t]{3}{*}{ Gender } & \multirow[t]{3}{*}{ Province } & \multicolumn{4}{|l|}{ Variables } & \multirow[t]{2}{*}{ Total (n) } & \multirow[t]{2}{*}{$x^{2}$} & \multirow[t]{2}{*}{$P$} \\
\hline & & \multicolumn{4}{|c|}{ Imported case $n(\%)$} & & & \\
\hline & & \multicolumn{2}{|l|}{ No } & \multicolumn{2}{|l|}{ Yes } & & & \\
\hline \multirow[t]{2}{*}{ Female } & Zhejiang & \multicolumn{2}{|l|}{$293(56.8 \%)$} & \multicolumn{2}{|l|}{$223(43.2 \%)$} & 516 & 2.022 & 0.155 \\
\hline & Henan & \multicolumn{2}{|c|}{$297(52.5 \%)$} & \multicolumn{2}{|l|}{$269(47.5 \%)$} & 566 & & \\
\hline \multirow[t]{4}{*}{ Male } & Zhejiang & \multicolumn{2}{|l|}{$266(51 \%)$} & \multicolumn{2}{|l|}{$256(49.0 \%)$} & 522 & 15.044 & $<0.001$ \\
\hline & Henan & \multicolumn{2}{|c|}{$266(39.7 \%)$} & \multicolumn{2}{|l|}{$404(60.3 \%)$} & 670 & & \\
\hline & & \multicolumn{4}{|l|}{ Age n (\%) } & & & \\
\hline & & $(0-18)$ & $(19-35)$ & $(36-59)$ & $(\geq 60)$ & & & \\
\hline \multirow[t]{2}{*}{ Female } & Zhejiang & $12(2.3 \%)$ & $104(19.5 \%)$ & $297(55.8 \%)$ & $119(22.4 \%)$ & 532 & \multirow[t]{2}{*}{13.856} & \multirow[t]{2}{*}{0.003} \\
\hline & Henan & $21(3.7 \%)$ & $148(25.8 \%)$ & $317(55.2 \%)$ & $88(15.3 \%)$ & 574 & & \\
\hline \multirow[t]{4}{*}{ Male } & Zhejiang & $18(3.3 \%)$ & $118(21.8 \%)$ & $307(56.6 \%)$ & $99(18.3 \%)$ & 542 & 17.225 & 0.001 \\
\hline & Henan & $32(4.7 \%)$ & $215(31.4 \%)$ & $335(49.0 \%)$ & $102(14.9 \%)$ & 684 & & \\
\hline & & Place of re & sidence n (\%) & & & & & \\
\hline & & Urban area & & Rural areas & & & & \\
\hline Female & Zhejiang & $430(81.9 \%$ & & $95(18.1 \%)$ & & 525 & 103.612 & $<0.001$ \\
\hline & Henan & $301(52.9 \%$ & & $268(47.1 \%)$ & & 569 & & \\
\hline Male & Zhejiang & $404(77.1 \%$ & & $120(22.9 \%)$ & & 524 & 111.466 & $<0.001$ \\
\hline & Henan & $321(47.1 \%$ & & $361(52.9 \%)$ & & 682 & & \\
\hline & & Hubei trav & history n (\%) & & & & & \\
\hline & & No & & Yes & & & & \\
\hline Female & Zhejiang & $271(60.5 \%$ & & $177(39.5 \%)$ & & 448 & 13.058 & $<0.001$ \\
\hline & Henan & $230(48.6 \%$ & & $243(51.4 \%)$ & & 473 & & \\
\hline Male & Zhejiang & $272(56.3 \%$ & & $211(43.7 \%)$ & & 483 & 36.591 & $<0.001$ \\
\hline & Henan & $213(37.6 \%$ & & $353(62.4 \%)$ & & 566 & & \\
\hline & & Wuhan tra & el history n (\% & & & & & \\
\hline & & No & & Yes & & & & \\
\hline Female & Zhejiang & $257(58.8 \%$ & & $180(41.2 \%)$ & & 437 & 5.364 & 0.021 \\
\hline & Henan & $242(51.2 \%$ & & $231(48.8 \%)$ & & 473 & & \\
\hline Male & Zhejiang & $257(53.9 \%$ & & $220(46.1 \%)$ & & 477 & 18.237 & $<0.001$ \\
\hline & Henan & $230(40.6 \%$ & & $336(59.4 \%)$ & & 566 & & \\
\hline & & Cases con & act history $\mathrm{n}$ ( & & & & & \\
\hline & & No & & Yes & & & & \\
\hline Female & Zhejiang & $37(12.2 \%)$ & & $266(87.8 \%)$ & & 303 & 1.429 & 0.232 \\
\hline & Henan & $27(9.2 \%)$ & & $267(90.8 \%)$ & & 294 & & \\
\hline Male & Zhejiang & $50(16.9 \%)$ & & $245(83.1 \%)$ & & 297 & 11.499 & 0.001 \\
\hline & Henan & $20(7.5 \%)$ & & $247(92.5 \%)$ & & 267 & & \\
\hline & & Time inter & al (day) & & & & $t$ & $P$ \\
\hline Female & Henan & $7.20 \pm 4.43$ & & & & & -1.285 & 0.199 \\
\hline & Zhejiang & $6.80 \pm 3.95$ & & & & & & \\
\hline Male & Henan & $7.48 \pm 4.01$ & & & & & -1.247 & 0.213 \\
\hline & Zhejiang & $7.11 \pm 4.23$ & & & & & & \\
\hline
\end{tabular}




\section{Comparison of laboratory-confirmed cases in Henan and Zhejiang after adjusting age}

Age is an important factor for COVID-19 related severity or deaths, and most of deaths are elderly based on data from Chinese center for disease control and prevention $(C D C)(6)$. Our results showed that the proportions of cases living in rural areas at different ages of Henan were higher than that in Zhejiang, with statistically significant differences $(P<0.05)$. Notably, the proportion of elderly cases living in rural areas in Henan is about 3 times of Zhejiang. $68.3 \%$ of $19 \sim$ 35 -year-old cases in Henan were imported, which was higher than that in Zhejiang and the difference was statistically significant $(P<0.001)$. The proportion of young cases (19 35-year-old) who had a Hubei $(69.8 \%)$ or Wuhan travel history $(68.2 \%)$ in Henan was higher than that in Zhejiang $(46.3 \%$, $45.7 \%)$ with statistically significant differences $(P<0.001) .55 .9 \%$ of cases at the age of $36 \sim 59$ had a Hubei travel history in Henan, which was higher than that in Zhejiang (43.8\%) with statistically significant difference $(P<0.001)$. The proportion of cases at $19 \sim 35(95.4 \%)$ or $36 \sim 59$-year-old $(91.5 \%)$ who had a case contact history in Henan was higher than that in Zhejiang $(84.5 \%, 82.4 \%)$ with statistically significant difference $(P<0.05)$. The time interval (from onset to diagnosis) of cases at $36 \sim 59$-year-old $(7.86 \pm 4.45)$ days in Henan was longer than that in Zhejiang $(7.16 \pm 4.12)$ days, with statistically significant difference $(P<0.05)$ (Table 5). These results indicate that more older cases lived in rural areas of Henan predicting a low medical level, may be related to high casefatality rate. Additionally, more young cases linked to an exposure of Hubei or Wuhan in Henan may be migrant workers, resulting in more imported cases at the same age level. The higher proportions of $36 \sim 59$-year-old cases with a case contact history or longer interval from onset to diagnosis may be related to high severe rate or deaths in Henan. 
Table 5

Comparison of laboratory-confirmed cases in Henan and Zhejiang after adjusting for age

\begin{tabular}{|c|c|c|c|c|c|c|}
\hline \multirow[t]{3}{*}{ Age } & \multirow[t]{3}{*}{ Province } & \multicolumn{2}{|l|}{ Variables } & Total (n) & $\chi^{2}$ & $P$ \\
\hline & & \multicolumn{5}{|l|}{ Gender n (\%) } \\
\hline & & Female & Male & & & \\
\hline \multirow[t]{2}{*}{$(0-18)$} & Zhejiang & $12(40.0 \%)$ & $18(60.0 \%)$ & 30 & \multirow[t]{2}{*}{0.001} & \multirow[t]{2}{*}{0.937} \\
\hline & Henan & $21(39.6 \%)$ & $32(60.4 \%)$ & 53 & & \\
\hline \multirow[t]{2}{*}{$(19-35)$} & Zhejiang & $104(46.8 \%)$ & $118(53.2 \%)$ & 222 & \multirow[t]{2}{*}{2.074} & \multirow[t]{2}{*}{0.150} \\
\hline & Henan & $148(40.8 \%)$ & $215(59.2 \%)$ & 363 & & \\
\hline \multirow[t]{2}{*}{$(36-59)$} & Zhejiang & $297(49.2 \%)$ & $307(50.8 \%)$ & 604 & \multirow[t]{2}{*}{0.038} & \multirow[t]{2}{*}{0.845} \\
\hline & Henan & $317(48.6 \%)$ & $335(51.4 \%)$ & 652 & & \\
\hline \multirow[t]{4}{*}{$(\geq 60)$} & Zhejiang & $119(54.6 \%)$ & $99(45.4 \%)$ & 218 & \multirow[t]{2}{*}{2.779} & \multirow[t]{2}{*}{0.096} \\
\hline & Henan & $88(46.3 \%)$ & $102(53.7 \%)$ & 190 & & \\
\hline & & \multicolumn{2}{|c|}{ Place of residence $\mathrm{n}(\%)$} & & & \\
\hline & & Urban areas & Rural areas & & & \\
\hline \multirow[t]{2}{*}{$(0-18)$} & Zhejiang & $24(85.7 \%)$ & $4(14.3 \%)$ & 28 & \multirow[t]{2}{*}{9.905} & \multirow[t]{2}{*}{0.002} \\
\hline & Henan & $26(50.0 \%)$ & $26(50.0 \%)$ & 52 & & \\
\hline \multirow[t]{2}{*}{$(19-35)$} & Zhejiang & $169(79.0 \%)$ & $45(21.0 \%)$ & 214 & \multirow[t]{2}{*}{65.899} & \multirow[t]{2}{*}{$<0.001$} \\
\hline & Henan & $160(44.3 \%)$ & $201(55.7 \%)$ & 361 & & \\
\hline \multirow[t]{2}{*}{$(36-59)$} & Zhejiang & $461(78.0 \%)$ & $130(22.0 \%)$ & 591 & \multirow[t]{2}{*}{95.993} & $<0.001$ \\
\hline & Henan & $331(51.2 \%)$ & $315(48.8 \%)$ & 646 & & \\
\hline$(\geq 60)$ & Zhejiang & 180(83.3\%) & $36(16.7 \%)$ & 216 & 38.552 & $<0.001$ \\
\hline & Henan & $104(55.0 \%)$ & $85(45.0 \%)$ & 189 & & \\
\hline & & Imported cas & n (\%) & & & \\
\hline & & No & Yes & & & \\
\hline$(0-18)$ & Zhejiang & 17(56.7\%) & $13(43.3 \%)$ & 30 & 0.008 & 0.928 \\
\hline & Henan & $30(57.7 \%)$ & $22(42.3 \%)$ & 52 & & \\
\hline$(19-35)$ & Zhejiang & $99(46.3 \%)$ & 115(53.7\%) & 214 & 12.233 & $<0.001$ \\
\hline & Henan & 113(31.7\%) & $244(68.3 \%)$ & 357 & & \\
\hline$(36-59)$ & Zhejiang & $288(49.7 \%)$ & $292(50.3 \%)$ & 580 & 2.927 & 0.087 \\
\hline & Henan & $286(44.8 \%)$ & $353(55.2 \%)$ & 639 & & \\
\hline$(\geq 60)$ & Zhejiang & $155(72.4 \%)$ & $59(27.6 \%)$ & 214 & 0.000 & 1.000 \\
\hline & Henan & $134(72.4 \%)$ & $51(27.6 \%)$ & 185 & & \\
\hline & & From Hubei $p$ & ovince n (\%) & & & \\
\hline & & No & Yes & & & \\
\hline$(0-18)$ & Zhejiang & $15(57.7 \%)$ & $11(42.3 \%)$ & 26 & 0.116 & 0.734 \\
\hline & Henan & $23(53.5 \%)$ & $20(46.5 \%)$ & 43 & & \\
\hline$(19-35)$ & Zhejiang & $109(53.7 \%)$ & $94(46.3 \%)$ & 203 & 28.686 & $<0.001$ \\
\hline & Henan & $96(30.2 \%)$ & $222(69.8 \%)$ & 318 & & \\
\hline$(36-59)$ & Zhejiang & $296(56.2 \%)$ & $231(43.8 \%)$ & 527 & 15.573 & $<0.001$ \\
\hline & Henan & $239(44.1 \%)$ & $303(55.9 \%)$ & 542 & & \\
\hline$(\geq 60)$ & Zhejiang & $123(70.3 \%)$ & $52(29.7 \%)$ & 175 & 1.401 & 0.237 \\
\hline & Henan & $85(63.9 \%)$ & $48(36.1 \%)$ & 133 & & \\
\hline
\end{tabular}




\begin{tabular}{|c|c|c|c|c|c|c|}
\hline \multirow[t]{3}{*}{ Age } & \multirow[t]{3}{*}{ Province } & \multicolumn{2}{|l|}{ Variables } & \multirow[t]{3}{*}{ Total $(n)$} & \multirow[t]{3}{*}{$\chi^{2}$} & \multirow[t]{3}{*}{$P$} \\
\hline & & \multicolumn{2}{|c|}{ From Wuhan city n (\%) } & & & \\
\hline & & No & Yes & & & \\
\hline \multirow[t]{2}{*}{$(0-18)$} & Zhejiang & $16(61.5 \%)$ & $10(38.5 \%)$ & 26 & \multirow[t]{2}{*}{0.011} & \multirow[t]{2}{*}{0.917} \\
\hline & Henan & $27(62.8 \%)$ & $16(37.2 \%)$ & 43 & & \\
\hline \multirow[t]{2}{*}{$(19-35)$} & Zhejiang & $107(54.3 \%)$ & $90(45.7 \%)$ & 197 & \multirow[t]{2}{*}{25.700} & \multirow[t]{2}{*}{$<0.001$} \\
\hline & Henan & 101(31.8\%) & $217(68.2 \%)$ & 217 & & \\
\hline \multirow[t]{2}{*}{$(36-59)$} & Zhejiang & $268(51.7 \%)$ & $250(48.3 \%)$ & 518 & \multirow[t]{2}{*}{2.517} & \multirow[t]{2}{*}{0.113} \\
\hline & Henan & $254(46.9 \%)$ & $288(53.1 \%)$ & 542 & & \\
\hline \multirow[t]{4}{*}{$(\geq 60)$} & Zhejiang & $123(71.1 \%)$ & $50(28.9 \%)$ & 173 & \multirow[t]{2}{*}{0.418} & \multirow[t]{2}{*}{0.518} \\
\hline & Henan & $90(67.7 \%)$ & $43(32.3 \%)$ & 133 & & \\
\hline & & \multicolumn{2}{|c|}{ Contact the case $\mathrm{n}(\%)$} & & & \\
\hline & & No & Yes & & & \\
\hline \multirow[t]{2}{*}{$(0-18)$} & Zhejiang & $2(10.0 \%)$ & $18(90.0 \%)$ & 20 & \multirow[t]{2}{*}{0.229} & \multirow[t]{2}{*}{$0.632^{*}$} \\
\hline & Henan & $1(2.9 \%)$ & $33(97.1 \%)$ & 34 & & \\
\hline \multirow[t]{2}{*}{$(19-35)$} & Zhejiang & $16(15.5 \%)$ & $87(84.5 \%)$ & 103 & \multirow[t]{2}{*}{8.012} & \multirow[t]{2}{*}{0.005} \\
\hline & Henan & $6(4.6 \%)$ & $124(95.4 \%)$ & 130 & & \\
\hline \multirow[t]{2}{*}{$(36-59)$} & Zhejiang & $56(17.6 \%)$ & $263(82.4 \%)$ & 319 & \multirow[t]{2}{*}{10.822} & \multirow[t]{2}{*}{0.001} \\
\hline & Henan & $24(8.5 \%)$ & $260(91.5 \%)$ & 284 & & \\
\hline \multirow[t]{3}{*}{$(\geq 60)$} & Zhejiang & $13(8.3 \%)$ & 143(91.7\%) & 156 & \multirow[t]{2}{*}{2.394} & 0.122 \\
\hline & Henan & $16(14.3 \%)$ & $96(85.7 \%)$ & 112 & & \\
\hline & & Time interva & day) & & $t$ & $P$ \\
\hline$(0-18)$ & Zhejiang & $4.70 \pm 2.64$ & & & -0.320 & 0.751 \\
\hline & Henan & $5.00 \pm 3.55$ & & & & \\
\hline$(19-35)$ & Zhejiang & $6.73 \pm 3.69$ & & & 0.166 & 0.868 \\
\hline & Henan & $6.67 \pm 3.43$ & & & & \\
\hline$(36-59)$ & Zhejiang & $7.16 \pm 4.12$ & & & -2.340 & 0.020 \\
\hline & Henan & $7.86 \pm 4.45$ & & & & \\
\hline$(\geq 60)$ & Zhejiang & $6.88 \pm 4.45$ & & & -0.942 & 0.347 \\
\hline & Henan & $7.39 \pm 3.35$ & & & & \\
\hline
\end{tabular}

\section{Comparison of laboratory-confirmed cases in Henan and Zhejiang after adjusting for imported cases}

Imported cases who have been infected at incubation, subclinical, or onset stage may lead to more severe cases or deaths, especially for the cases from Wuhan or Hubei. Our results showed $60.0 \%$ of imported cases in Henan are male, which was higher than that in Zhejiang (53.4\%), with statistically significant difference $(P<0.05)$. Among non-imported cases, there was no difference in gender distribution between the two provinces $(P>0.05)$. There were also differences in age distribution in imported cases between Henan and Zhejiang $(P<0.001)$, and more imported cases with $19 \sim 35$-year-old in Henan $(P<0.001)$ (Additional file 1: Table 1). Among imported cases, the proportion of cases having a Hubei travel history in Henan (90.1\%) was higher than that in Zhejiang $(81.2 \%)$ with a statistically significant difference $(P<0.001)$. Among non-imported cases, there was no statistically significant difference in visiting Hubei $(P>$ 0.05). Among imported cases or non-imported cases, higher proportions of cases in Henan came from rural areas, relative to Zhejiang with statistically significant differences $(P<0.001)$. There was no significant difference between the two provinces about the imported and non-imported cases having a Wuhan travel history $(P>0.05)$. Among imported cases, $96.9 \%$ of cases having a case contact history in Henan, which were higher than that in Zhejiang $(23.2 \%)$ with statistically significant difference $(P<0.001)$. Among non-imported cases, $100.0 \%$ of cases having a history of contact with cases in Zhejiang, which was higher than that in Henan (89.9\%), and the difference was statistically significant $(P<0.001)$ (Table 6). Together, our results indicate that higher proportions of imported cases are male or have a Hubei travel history, or live in rural areas. As mentioned above, imported cases from Hubei lived in rural areas may be migrant workers. 
Table 6

Comparison of laboratory-confirmed cases in Henan and Zhejiang after adjusting for imported case

\begin{tabular}{|c|c|c|c|c|c|c|c|c|}
\hline \multirow[t]{3}{*}{ Imported case } & \multirow[t]{3}{*}{ Province } & \multicolumn{4}{|l|}{ Variables } & Total (n) & $x^{2}$ & $P$ \\
\hline & & \multicolumn{7}{|c|}{ Gender n (\%) } \\
\hline & & \multicolumn{2}{|l|}{ Female } & \multicolumn{5}{|l|}{ Male } \\
\hline \multirow[t]{2}{*}{ No } & Zhejiang & \multicolumn{2}{|l|}{$293(52.4 \%)$} & \multicolumn{2}{|l|}{$266(47.6 \%)$} & 559 & \multirow[t]{2}{*}{0.013} & \multirow[t]{2}{*}{0.910} \\
\hline & Henan & $297(52.8 \%)$ & & $266(47.2 \%)$ & & 563 & & \\
\hline \multirow[t]{4}{*}{ Yes } & Zhejiang & \multicolumn{2}{|l|}{$223(46.6 \%)$} & \multicolumn{2}{|l|}{$256(53.4 \%)$} & 479 & \multirow[t]{2}{*}{4.959} & \multirow[t]{2}{*}{0.026} \\
\hline & Henan & $269(40.0 \%)$ & & $404(60.0 \%)$ & & 673 & & \\
\hline & & \multicolumn{4}{|l|}{ Age n (\%) } & & & \\
\hline & & $(0-18)$ & $(19-35)$ & $(36-59)$ & $(\geq 60)$ & & & \\
\hline \multirow[t]{2}{*}{ No } & Zhejiang & $17(3 \%)$ & $99(17.7 \%)$ & $288(51.5 \%)$ & $155(27.7 \%)$ & 559 & \multirow[t]{2}{*}{6.039} & \multirow[t]{2}{*}{0.110} \\
\hline & Henan & $30(5.3 \%)$ & $113(20.1 \%)$ & $286(50.8 \%)$ & $134(23.8 \%)$ & 563 & & \\
\hline \multirow[t]{4}{*}{ Yes } & Zhejiang & $13(2.7 \%)$ & $115(24 \%)$ & $292(61 \%)$ & $59(12.3 \%)$ & 479 & \multirow[t]{2}{*}{23.930} & \multirow[t]{2}{*}{$<0.001$} \\
\hline & Henan & $22(3.3 \%)$ & $244(36.4 \%)$ & $353(52.7 \%)$ & $51(7.6 \%)$ & 670 & & \\
\hline & & \multicolumn{4}{|c|}{ Place of residence $\mathrm{n}(\%)$} & & & \\
\hline & & Urban area & & Rural areas & & & & \\
\hline No & Zhejiang & $430(77.3 \%)$ & & $126(22.7 \%)$ & & 556 & 50.988 & $<0.001$ \\
\hline & Henan & $322(57.3 \%)$ & & $240(42.7 \%)$ & & 562 & & \\
\hline Yes & Zhejiang & $375(81.3 \%)$ & & $86(18.7 \%)$ & & 461 & 157.418 & $<0.001$ \\
\hline & Henan & $292(44.0 \%)$ & & $372(56.0 \%)$ & & 664 & & \\
\hline & & From Hube & province $n$ (\% & & & & & \\
\hline & & No & & Yes & & & & \\
\hline No & Zhejiang & $452(100.0 \%$ & & $0(0.0 \%)$ & & 452 & 1.747 & $0.186^{*}$ \\
\hline & Henan & $373(99.2 \%)$ & & $3(0.8 \%)$ & & 376 & & \\
\hline Yes & Zhejiang & 91(18.8\%) & & $392(81.2 \%)$ & & 483 & 18.865 & $<0.001$ \\
\hline & Henan & $65(9.9 \%)$ & & $592(90.1 \%)$ & & 657 & & \\
\hline & & From Wuha & n city n (\%) & & & & & \\
\hline & & No & & Yes & & & & \\
\hline No & Zhejiang & $447(99.3 \%)$ & & $3(0.7$ & & 450 & 0.000 & $1.000 *$ \\
\hline & Henan & $373(99.2 \%)$ & & $3(0.8$ & & 376 & & \\
\hline Yes & Zhejiang & $67(14.3 \%)$ & & 401( & $5.7 \%)$ & 468 & 0.000 & 0.997 \\
\hline & Henan & $94(14.3 \%)$ & & 563 & $5.7 \%)$ & 657 & & \\
\hline & & Contact the & case n (\%) & & & & & \\
\hline & & No & & Yes & & & & \\
\hline No & Zhejiang & $0(0.0 \%)$ & & 477( & $0.0 \%)$ & 477 & 50.799 & $<0.001$ \\
\hline & Henan & $43(10.1 \%)$ & & 381( & $.9 \%)$ & 424 & & \\
\hline Yes & Zhejiang & $86(76.8 \%)$ & & $26(2$ & $2 \%)$ & 112 & 139.936 & $<0.001$ \\
\hline & Henan & $4(3.1 \%)$ & & 126( & $5.9 \%)$ & 130 & & \\
\hline & & Time interv & al (day) & & & & $t$ & $P$ \\
\hline No & Zhejiang & $6.87 \pm 4.34$ & & & & & -1.620 & 0.106 \\
\hline & Henan & $7.36 \pm 4.38$ & & & & & & \\
\hline Yes & Zhejiang & $7.01 \pm 3.67$ & & & & & -0.829 & 0.408 \\
\hline
\end{tabular}

Page 12/21 


\begin{tabular}{|c|c|c|c|c|}
\hline Imported case & Province & Variables & Total (n) & $P$ \\
\hline & Henan & $7.25 \pm 3.97$ & & \\
\hline
\end{tabular}

\section{Comparison of laboratory-confirmed cases in Henan and Zhejiang after adjusting place of residence}

There is a big urban-rural gap in health resource allocation of public medical institutions in China(10), implying cases from rural areas may have a higher risk of developing severity or death. Our results showed that there was no statistical difference in gender after the stratification $(P>0.05)$. There were statistical differences in different age groups whatever rural or urban areas $(P<0.05)$. 21.6\% of cases living in urban areas were $\geq 60$-year-old in Zhejiang, which was higher than that in Henan (16.7\%) with statistically significant difference $(P<0.05)$ (Additional file 1: Table 1). Consistent to early analysis, more cases at the age of $36 \sim 59$-year-old or $\geq 60$-year-old lived in rural areas in Henan, relative to Zhejiang, and the differences were statistically significant $(P<0.001)$. 60.8\% of rural cases were imported cases in Henan, which were higher than that in Zhejiang (40.6\%) with statistically significant difference $(P<0.001)$. Those who lived in rural areas of Henan with Hubei $(66.7 \%)$ or Wuhan travel history $(65.2 \%)$ were more than that in Zhejiang $(35.0 \%, 38.7 \%)$, and the differences were statistically significant $(P<0.001)$. There were $91.9 \%$ of cases in urban areas having a contact history, which were higher than that in Zhejiang $85.5 \%$ with statistically significant differences $(P<0.05)$. The difference of time interval from onset to diagnosis between two provinces had no statistically significance $(P>0.05)$, but the time interval in rural areas of Henan was 0.8 day longer than Zhejiang (Table 7). Together, our data indicate that a higher proportion of elderly cases lived in rural areas of Henan, and more young cases with epidemiological history in Henan come from rural areas, relative to Zhejiang. 
Table 7

Comparison of laboratory-confirmed cases in Henan and Zhejiang after adjusting place of residence

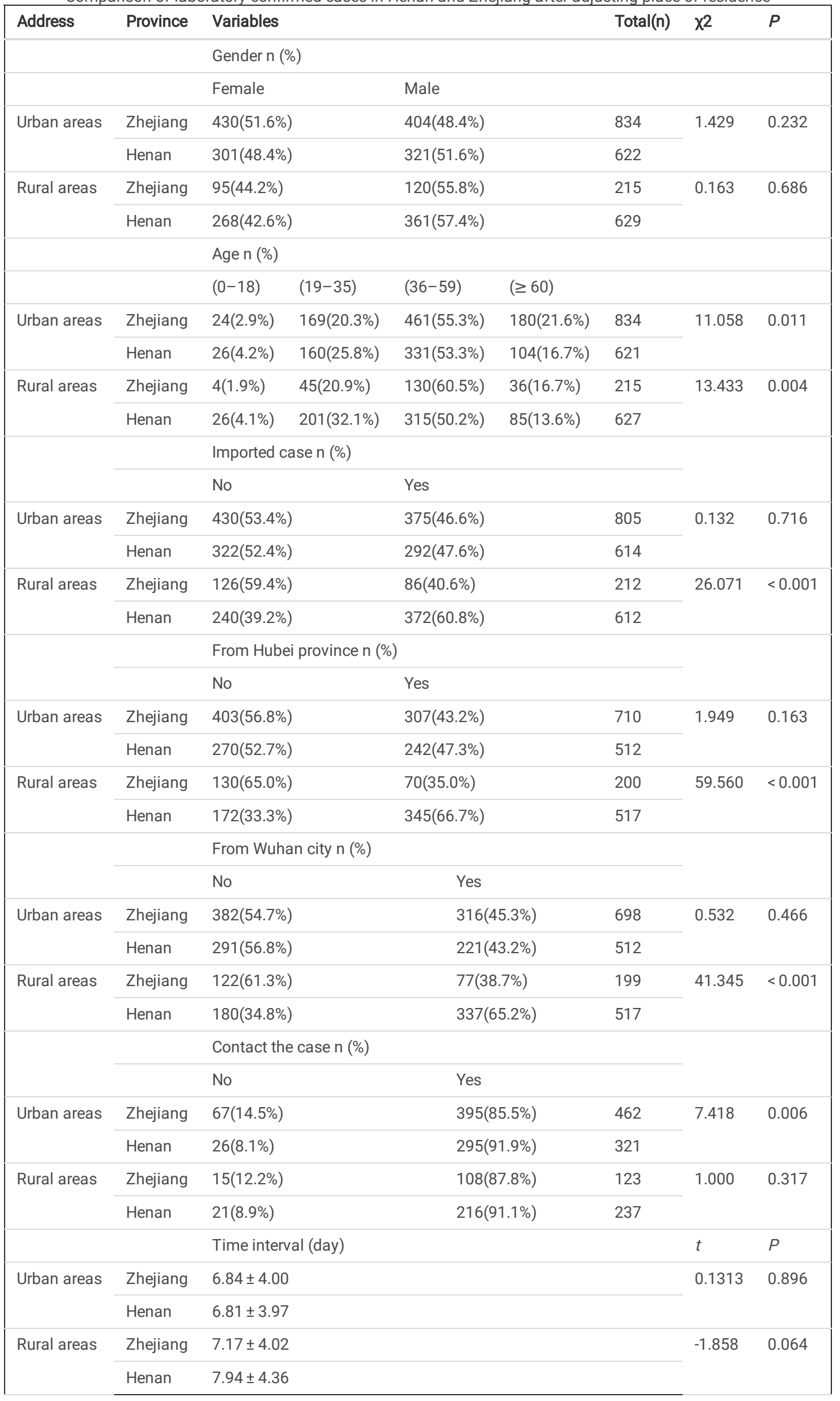

Page $14 / 21$ 


\section{Comparison of designated hospitals for COVID-19 treatment in Henan and Zhejiang}

Generally, a higher-grade hospital will be beneficial for COVID-19 treatment. 147 and 100 designated treatment hospitals were announced by Henan and Zhejiang Heath Commissions, respectively (Figure.5A). Most of designated treatment hospitals in Henan were grade 2A (103), while designated treatment hospitals in Zhejiang were mainly from 3A (25), 3B (32) and 2A (37). In order to further explore whether there is really a difference in the level of treatment for those confirmed cases, we analyzed the medical level of the specific hospitals where the patients were treated. Together, we found that the patients in Zhejiang were indeed treated in higher-grade hospitals, and this difference was statistically significant $(P<0.001)$ (Figure.5B and Figure.5C).

\section{Discussion}

Currently, COVID-19 still remains a big challenge for China and global public health. Even though most of cases are asymptomatic or mild, a large number of severe cases have cost a lot of medical resources and pose a tremendous pressure on clinical treatment. Nowadays, few of cases continue to die every day which cause serious panic to society. Outside Hubei, the case-fatality rate of most provinces such as Zhejiang keeps an extremely low level, while some of provinces such as Henan and Heilongjiang far exceed national average level. Recent studies have indicated that gender (male), age (elder), and physical health (underlying basic disorders) are closely related to the adverse outcomes of the disease $(4,6)$. To deep understand factors behind COVID- 19 related severity or mortality, we compared variables of initial symptoms, gender, age, place of residence, epidemiological history, time interval from onset to diagnosis and designated hospitals for COVID-19 treatment in Henan and Zhejiang.

First, we found that higher severe rate at early epidemic phase, lower cure rate and higher number of critical cases were found in Henan, relative to Zhejiang. It has been reported that most of deaths come from severe and critical cases(7), and lower cure rate may associate with medical level of Henan. Features of COVID-19 related deaths suggest that most of deaths come from elderly and male cases and those who have underlying basic diseases or a history of contact with cases, or live in rural areas. Previous study also indicated that elderly patients and those who have potential chronic diseases such as respiratory diseases, cardiovascular diseases, diabetes, hypertension and cancer $(7,11)$. Management of elderly patients, especially those with chronic diseases should be concerned in clinical practice(12). SARS-CoV-2, the pathogen of COVID-19, hijacks human angiotensin converting enzyme 2 (ACE2) receptor for cell entry, the expression of ACE2 involves with the severity of COVID-19(13,14). Male persons with smoking showed higher ACE2 expression in lungs(9), which may explain the higher proportion of deaths in male population. Fever is an important predictor for the severity of infectious diseases(15, 16). Compare to Zhejiang, Henan has a higher proportion of cases with fever, which may support the higher severe rate of Henan in the early epidemic phase. Several studies have reported that asymptomatic patients can shed virus, but whether asymptomatic infection is contagious remains controversial $(6,17,18)$. It is now generally accepted that asymptomatic infection is weak, indicating Zhejiang with a higher proportion of asymptomatic infection, may have more mild patients compared to Henan.

Next, we conducted stratified analysis of the data from these two provinces. Our findings suggest that most of imported cases in rural areas of Henan come from male young persons who have a Hubei or Wuhan travel history, or directly contact with cases. Henan is the largest labor export province in China, and the labor output is nearly 13 million in 2018(19). Therefore, we speculate this group of infected persons are migrant workers and return to Henan before the Spring Festival. The medical level and health literacy in rural areas are always lower than urban areas $(20,21)$. These group of infected persons predict to cause their family members infection, which is difficult to control. Therefore, Chinese governments at all levels adopted containment strategies at early phase of epidemic, and had been demonstrated to be effective.

Overall, the average age of cases in Zhejiang was higher than that in Henan, and most cases are middle-aged and elderly infected persons, because aging population in Zhejiang is more serious than in Henan $(22,23)$. However, the case-fatality rate was $0.0 \%$ until February 19 , which was contrary to our original inference. Our further analysis indicates that most of elderly cases in Zhejiang live in urban areas, while the higher proportion of elderly cases in Henan live in rural areas with a longer time interval, and the time interval in rural areas of Henan is also longer relative to Zhejiang. The level of urbanization in Zhejiang is $68.9 \%$, while Henan is only $51.71 \%(24,25)$. There is a big urban-rural gap in health resource allocation of public medical institutions in China(10), Zhejiang has higher-grade of hospitals for COVID-19 treatment, which may explain a higher cure rate and lower severe rate compared to Henan. In the previous battles against SARS and H7N9 $(26,27)$, advanced treatment strategy of severe respiratory diseases was also well established in Zhejiang, and a lower number of critical cases was observed in Zhejiang. The longer interval from onset to diagnosis can also affect the development of disease in elderly infected persons in Henan. As for above reasons, we think elderly cases living in rural areas may be responsible for the higher severe rate and number of critical cases, which further cause more deaths in Henan. At last, as lack of detail information of the reported cases from Henan and Zhejiang, there are certain limitations to our study.

\section{Conclusion}

In summary, our study found that higher proportions of patients with fever, imported male cases with a contact history, and elderly cases with a longer time interval from onset to diagnosis who live in rural areas, and the lower proportion of asymptomatic infection and the lower-grade of hospitals for COVID-19 treatment might be related to COVID-19 severity and mortality in Henan. Thus, our findings suggest that initial symptoms, age, place of residence, gender, the time interval from onset to diagnosis and the grade of designated hospital for patient's treatment may be potential factors behind COVID-19 related severity or mortality.

\section{Declarations}

\section{Ethics approval and Consent to participate}


The data used in this study was completely from public data released by the government, so the ethics approval and consent to participate were not applicable.

\section{Consent for publication}

Not applicable.

\section{Availability of data and materials}

All data generated and analyzed during this study are included in this published article and its supplementary information files.The details are available from the following official websites: National Health Committee of the People's Republic of China (http://www.nhc.gov.cn/), Health Commission of Henan Province (http://www.hnwsjsw.gov.cn/Template/fangkong.shtml), Health Commission of Zhejiang Province (http://www.zjwjw.gov.cn/col/col1202101/index.html) and other official websites of urban areas.

\section{Competing interests}

The authors declare no conflict of interests.

\section{Funding}

This work was funded by the National Science and Technology Specific Projects (NO.2018ZX10301407); National Natural Science Foundation of China (N0.81172740 and N0.81573205); Project founded by China Postdoctoral Science Foundation (NO.2019M662543).

\section{Authors' contributions}

Wangquan Ji: Research concept and design, Supervision, Data analysis and interpretation, Data acquisition and verification, Writing original draft. Ruonan Liang: Data acquisition and verification, Data analysis. Peiyu Zhu: Data acquisition and verification, Data analysis. Haiyan Yang: Review and modify. Shuaiyin Chen: Review and modify. Weiguo Zhang: Review and modify. Guangcai Duan and Yuefei Jin: Conceptualization, Funding acquisition, Writing, Review \& editing, Final approval of the version to be submitted.

\section{Acknowledgements}

Not applicable.

\section{References}

1. Zhu N, Zhang D, Wang W, Li X, Yang B, Song J, et al. A Novel Coronavirus from Patients with Pneumonia in China, 2019. New England Journal of Medicine. 2020.

2. National Health Commission of People's Republic of China. An Update of Novel Coronavirus Pneumonia Outbreak as of 24:00 on March 09.

3. Li Q, Guan X, Wu P, Wang X, Zhou L, Tong Y, et al. Early Transmission Dynamics in Wuhan, China, of Novel Coronavirus-Infected Pneumonia. New England Journal of Medicine. 2020.

4. Huang C, Wang Y, Li X, Ren L, Zhao J, Hu Y, et al. Clinical features of patients infected with 2019 novel coronavirus in Wuhan, China. Lancet (London, England). 2020 Feb 15;395(10223):497-506. PubMed PMID: 31986264. Epub 2020/01/28. eng.

5. Gorbalenya AE, Baker SC, Baric RS, de Groot RJ, Drosten C, Gulyaeva AA, et al. <em>Severe acute respiratory syndrome-related coronavirus</em>: The species and its viruses - a statement of the Coronavirus Study Group. bioRxiv. 2020:2020.02.07.937862.

6. [The epidemiological characteristics of an outbreak of 2019 novel coronavirus diseases (COVID-19) in China]. Zhonghua liu xing bing xue za zhi = Zhonghua liuxingbingxue zazhi. 2020 Feb 17;41(2):145-51. PubMed PMID: 32064853. Epub 2020/02/18. chi.

7. Yang X, Yu Y, Xu J, Shu H, Xia J, Liu H, et al. Clinical course and outcomes of critically ill patients with SARS-CoV-2 pneumonia in Wuhan, China: a singlecentered, retrospective, observational study. The Lancet Respiratory medicine. 2020 Feb 24. PubMed PMID: 32105632. Epub 2020/02/28. eng.

8. National Health Commission of People's Republic of China. An Update of Novel Coronavirus Pneumonia Outbreak as of 24:00 on February 19.

9. Cai G. Bulk and single-cell transcriptomics identify tobacco-use disparity in lung gene expression of ACE2, the receptor of 2019-nCov. medRxiv. 2020:2020.02.05.20020107.

10. Li H, Chang GH. Disparity in Health Resource Allocation Between Rural and Urban Areas in China: Is It Getting Worse? Chinese Economy.41(6):45-55.

11. Liang W, Guan W, Chen R, Wang W, Li J, Xu K, et al. Cancer patients in SARS-CoV-2 infection: a nationwide analysis in China. The Lancet Oncology. 2020 Feb 14. PubMed PMID: 32066541. Epub 2020/02/19. eng.

12. Xu X-W, Wu X-X, Jiang X-G, Xu K-J, Ying L-J, Ma C-L, et al. Clinical findings in a group of patients infected with the 2019 novel coronavirus (SARS-Cov-2) outside of Wuhan, China: retrospective case series. BMJ. 2020.

13. Zhou P, Yang XL, Wang XG, Hu B, Zhang L, Zhang W, et al. A pneumonia outbreak associated with a new coronavirus of probable bat origin. Nature. 2020 Feb 3. PubMed PMID: 32015507. Epub 2020/02/06. eng.

14. Xin Zou KC, Jiawei Zou, Peiyi Han, Jie Hao, Zeguang Han. The single-cell RNA-seq data analysis on the receptor ACE2 expression reveals the potential risk of different human organs vulnerable to Wuhan 2019-nCoV infection. Front Med.0- 
15. Qin L, Dang D, Wang X, Zhang R, Feng H, Ren J, et al. Identification of immune and metabolic predictors of severe hand-foot-mouth disease. PloS one. 2019;14(5):e0216993. PubMed PMID: 31120941. Pubmed Central PMCID: PMC6532886. Epub 2019/05/24. eng.

16. Ashman RB, Müllbacher A. Infectious disease, fever, and the immune response. Immunology Today. 1984;5(9):268-71.

17. Rothe C, Schunk M, Sothmann P, Bretzel G, Froeschl G, Wallrauch C, et al. Transmission of 2019-nCoV Infection from an Asymptomatic Contact in Germany. New England Journal of Medicine. 2020.

18. Bai Y, Yao L, Wei T, Tian F, Jin DY, Chen L, et al. Presumed Asymptomatic Carrier Transmission of COVID-19. Jama. 2020 Feb 21. PubMed PMID: 32083643. Pubmed Central PMCID: PMC7042844. Epub 2020/02/23. eng.

19. Qiqi A. The Problem of Export of Labor Services in Henan Province and Its Countermeasures. jiangsu commercial forum. 2018.

20. Karlsson EL. Care groups and primary health care in rural areas. Israel Journal of Medical Sciences. 1983;19(8):731-3.

21. Pop OM, Brinzaniuc A, Sirlincan EO, Baba CO, Chereches RM. Assessing health literacy in rural settings: a pilot study in rural areas of Cluj County, Romania. Glob Health Promot.20(4):35-43.

22. Henan Provincial People's Government. Henan Province actively responds to the population aging "Respect for the Elderly" Press Conference.

23. Zhejiang Provincial People's Government. Reply of Zhejiang Provincial Market Supervision Bureau on Proposal No. 17 of the Second Session of the 13th National People's Congress.

24. Henan Provincial People's Government. Population Profile of Henan Province.

25. Zhejiang Provincial People's Government. Bulletin of main data of 1\% population sampling survey in Zhejiang Province in 2018.

26. Gao HN, Lu HZ, Cao B, Du B, Shang H, Gan JH, et al. Clinical findings in 111 cases of influenza A (H7N9) virus infection. The New England journal of medicine. 2013 Jun 13;368(24):2277-85. PubMed PMID: 23697469. Epub 2013/05/24. eng.

27. Gao H, Yao H, Yang S, Li L. From SARS to MERS: evidence and speculation. Frontiers of medicine. 2016 Dec;10(4):377-82. PubMed PMID: 27726088. Epub 2016/10/12. eng.

\section{Figures}

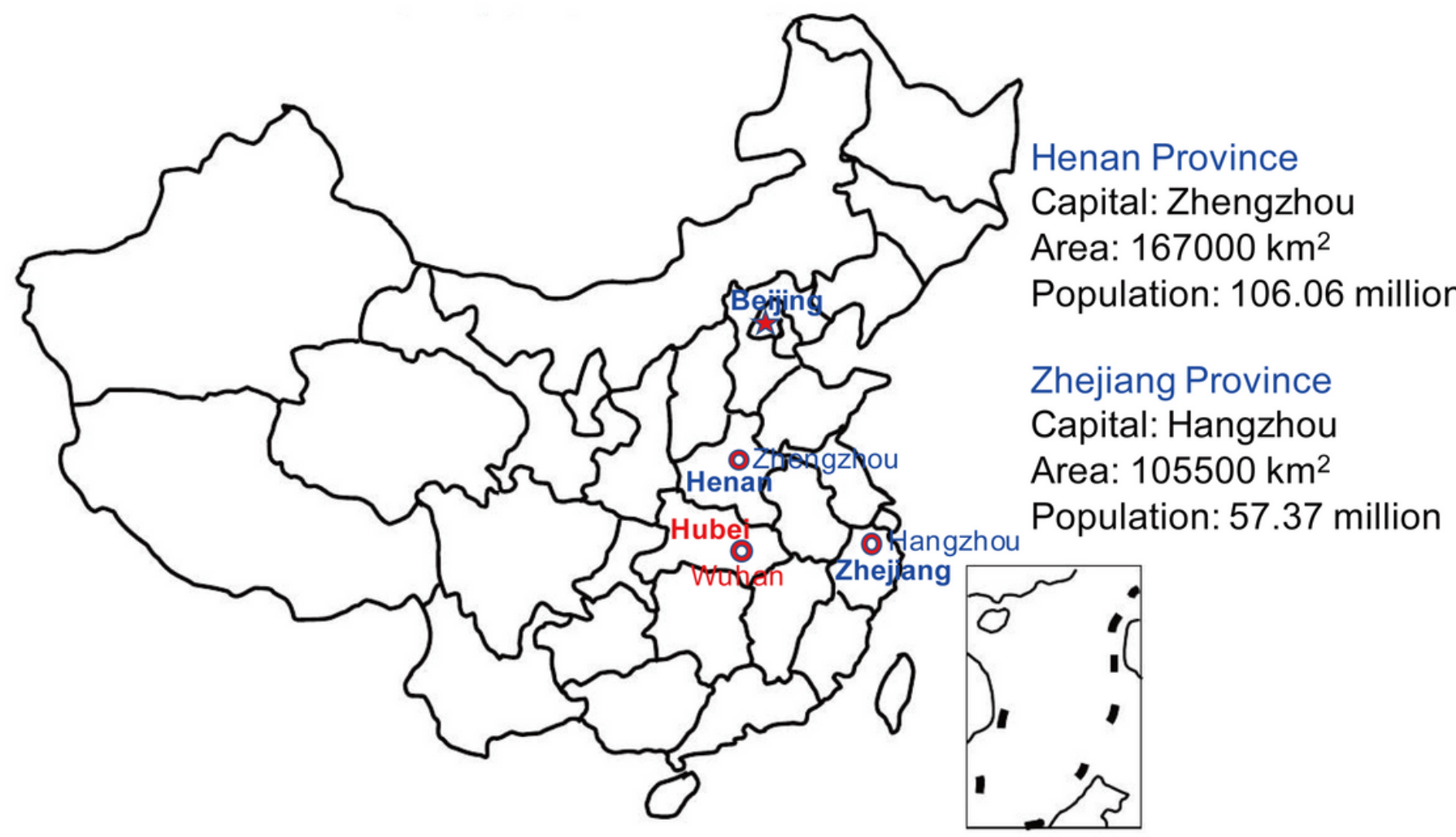

Figure 1

Geographic location of Henan Province and Zhejiang Province More information is available at http://www.henan.gov.cn/jchn/ and http://www.zj.gov.cn/col/col1544731/index.html. Note: The designations employed and the presentation of the material on this map do not imply the 
expression of any opinion whatsoever on the part of Research Square concerning the legal status of any country, territory, city or area or of its authorities, or concerning the delimitation of its frontiers or boundaries. This map has been provided by the authors.

A

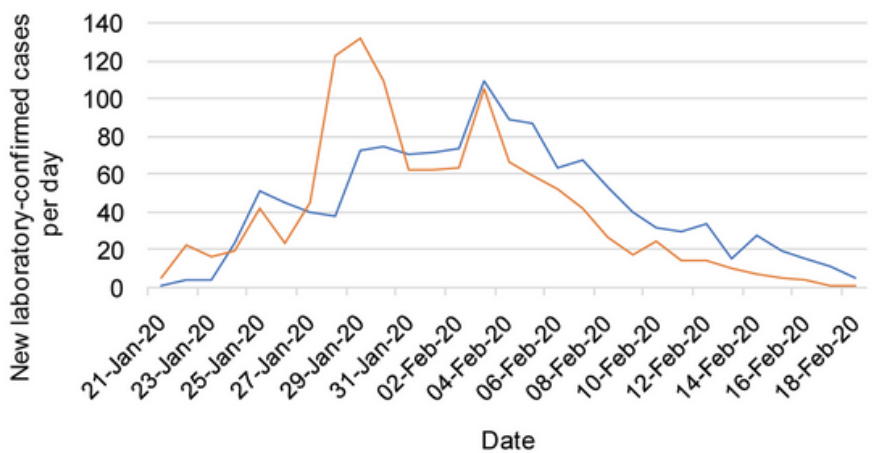

- Henan —Zhejiang

C

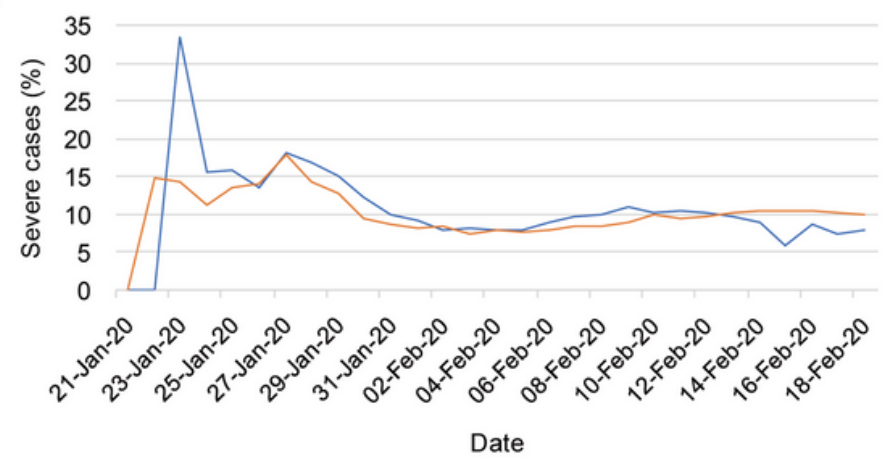

- Henan - Zhejiang
B

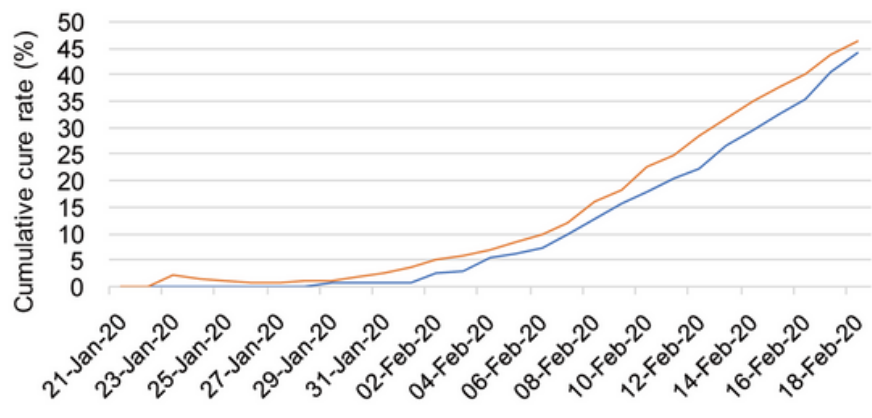

Date
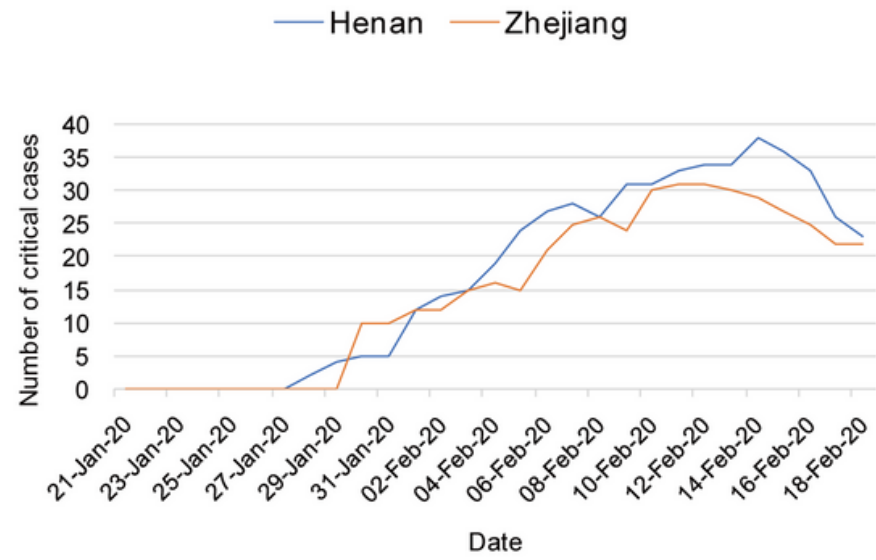

- Henan - Zhejiang

Figure 3

An overview of COVID-19 epidemic in Henan and Zhejiang Province (A): New laboratory-confirmed cases per day in Henan and Zhejiang; (B): The cumulative cure rate of Henan and Zhejiang; (C): Severe rate of Henan and Zhejiang; (D): The number of critical cases in Henan and Zhejiang. 


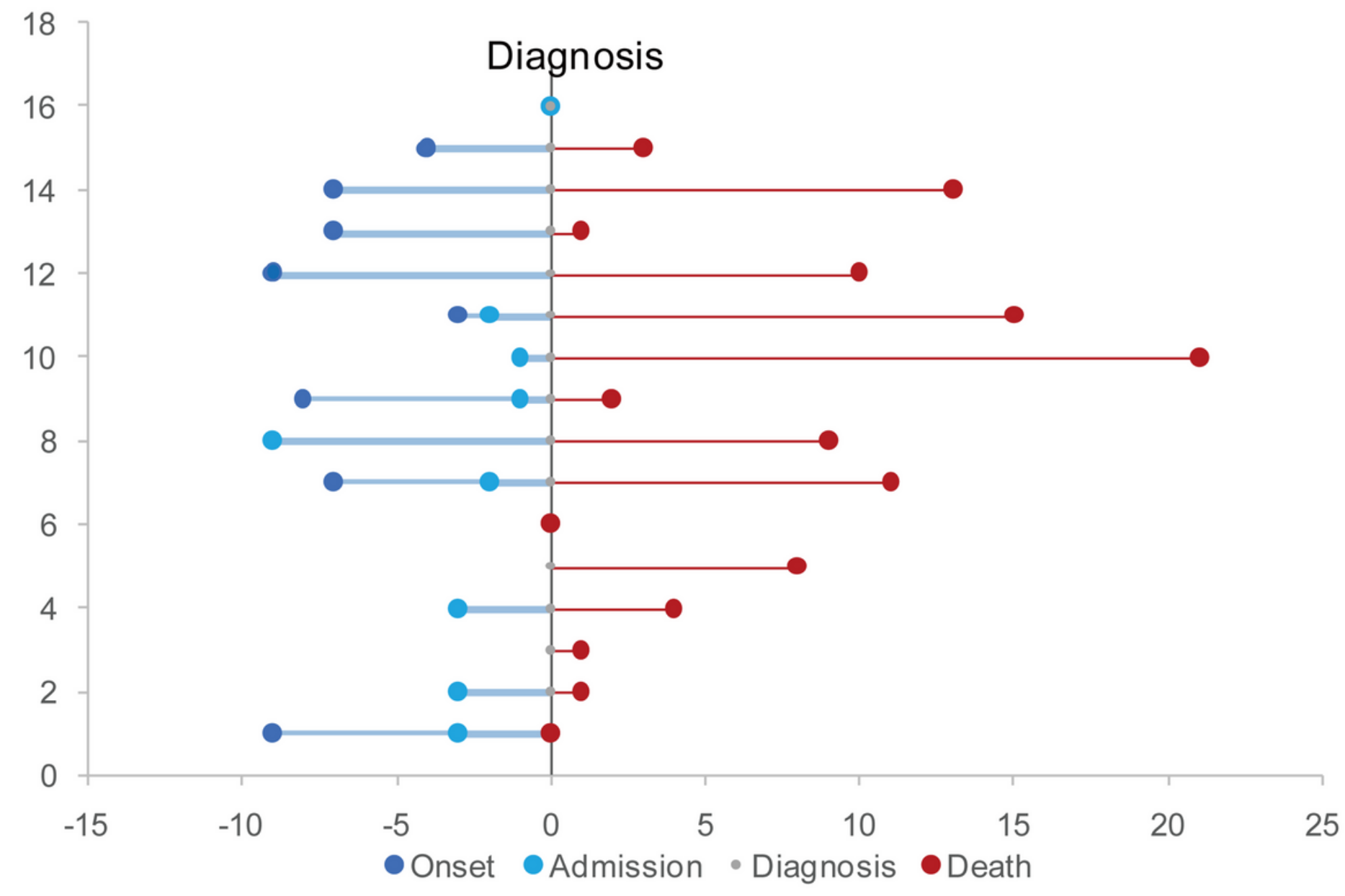

Figure 5

The time points of onset, admission, diagnosis and death in 16 death cases. The time points of onset, admission, diagnosis and death in 16 death cases with known information in Henan. 


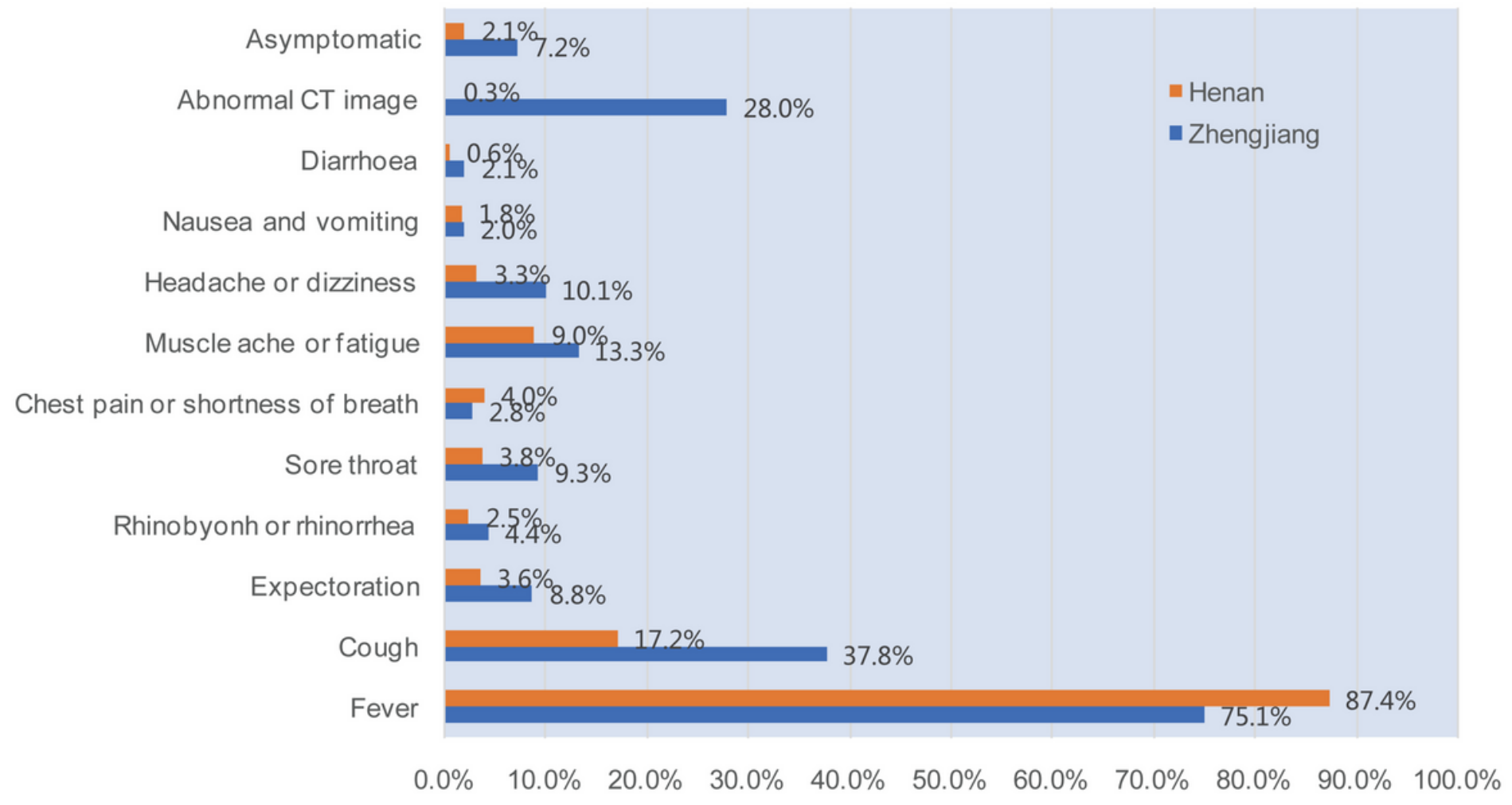

\section{Figure 7}

Initial symptoms of laboratory-confirmed cases in Henan and Zhejiang Province 939 cases in Zhejiang province and 676 cases in Henan province with the data of initial symptoms were collected and analyzed in this study. 
A

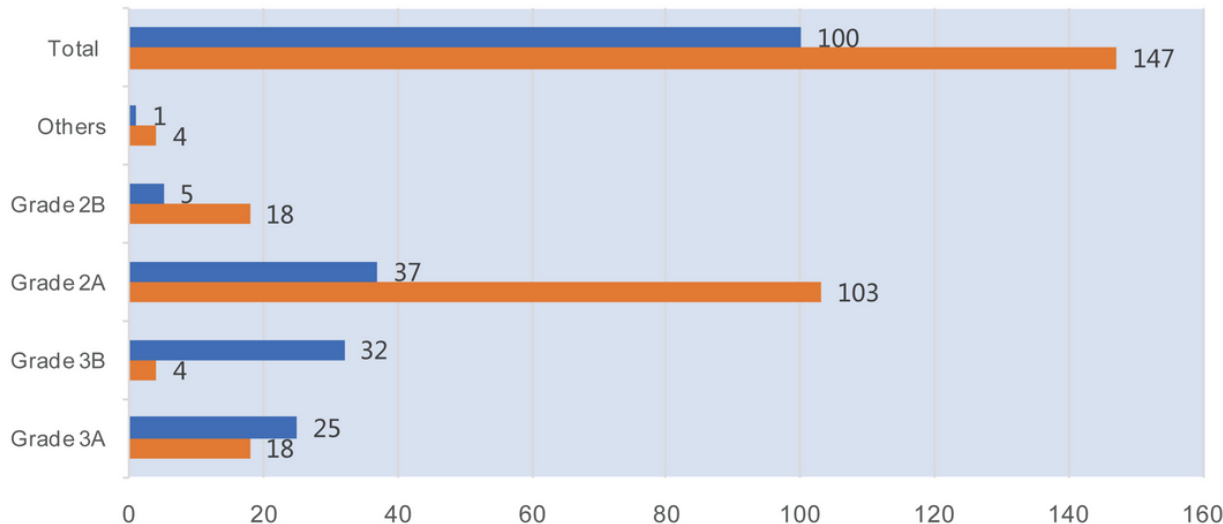

B

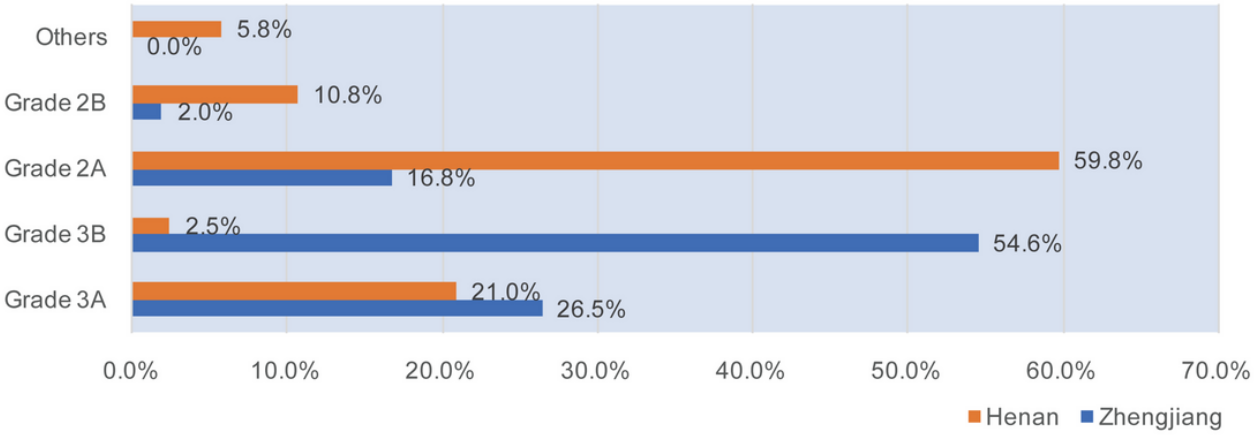

C

Comparison of the grade of hospitals for patients' treatment between Henan and Zhejiang

\begin{tabular}{|c|c|c|c|c|c|c|c|c|c|}
\hline \multirow{2}{*}{ Province } & \multicolumn{5}{|c|}{ Number of cases } & \multirow[b]{2}{*}{ Total } & \multirow{2}{*}{$\begin{array}{l}\text { Average } \\
\text { rank }\end{array}$} & \multirow{2}{*}{$Z$} & \multirow{2}{*}{$P$} \\
\hline & $3 A$ & $3 \mathrm{~B}$ & $2 A$ & $2 B$ & Others & & & & \\
\hline Henan & 256 & 31 & 729 & 132 & 71 & 1219 & 876.24 & & \\
\hline Zhejiang & 274 & 565 & 174 & 21 & 0 & 1034 & 1422.62 & -20.897 & $<0.001$ \\
\hline
\end{tabular}

\section{Figure 9}

The grade of designated hospitals for COVD-19 treatment in Henan and Zhejiang 1034 cases in Zhejiang and 1219 cases in Henan with the data of treatment hospital were collected and compared in this study. (A) The comparison of the grade of designated hospitals between Henan and Zhejiang. (B) The distribution of cases in different grades of designated hospitals in Henan and Zhejiang. (C) The comparison of the grade of designated hospitals for patients treatment between Henan and Zhejiang.

\section{Supplementary Files}

This is a list of supplementary files associated with this preprint. Click to download.

- Additionalfile1.pdf

- Additionalfile1.pdf 